\title{
Clinical performance and survival rate of mandibular overdentures retained by two implants: a case series
}

\section{BASIC RESEARCH}

\author{
Henriques I. ${ }^{1}$; Pinto, C. ${ }^{1}$; Lagrange, J.2; Fialho, J ${ }^{3}$; Chen A. ; Francisco, H. ${ }^{4}$ \\ Caramês, J. ${ }^{5}$
}

1 Resident at Postgraduation Course in Oral Surgery, University of Lisbon Faculty of Dental Medicine

2 Invited Assistant at the Postgraduate program in Oral Surgery and Implantology, University of Lisbon Faculty of Dental Medicine ${ }^{3}$ Adjunct Professor, Instituto Politécnico de Viseu (IPV) e CI\&DETS (Centro de Estudos em Educação, Tecnologias e Saúde) ${ }^{4}$ Assistant Professor at the Oral Surgery and Implant Dentistry Department at University of Lisbon Faculty of Dental Medicine

5 Full Professor at the Oral Surgery and Implant Dentistry Department at University of Lisbon Faculty of Dental Medicine

\section{Abstract}

BACKGROUND: Implant therapy with two implants placed in the edentulous mandible to support a removable overdenture has been considered a safe and predictable treatment modality. It shows positive results in terms of increasing prothesis stability and retention and consequently improving the chewing function and patient's quality of life.

PURPOSE: The aim of this study was to describe a series of cases of mandibular overdentures retained by two unsplited implants and evaluate their survival rate. MATERIALS AND METHODS: A retrospective case series of 13 patients rehabilitated with mandibular overdentures retained by two implants, at a Postgraduation Clinic in Lisbon between October 2017 and April 2019, was performed. Demographic data (age and gender), patient motivation and status of the prior dentition were described. The following aspects related to the rehabilitation were also registered: brand, number, diameter and length of the implants, prosthetic restoration (fabrication of a new immediate prothesis or adaptation of the old one), number of appointments after loading, complications, necessity of rebase, frequency of changing rubers, among others.

The clinical protocol consisted of 2 implants placed at 32 and 42 positions to retain a mandibular overdenture with the connection of Locator attachments (torqued to $25 \mathrm{Ncm}$ ). Descriptive statistical analysis was performed.

RESULTS: The sample was composed by 13 patients (53,8\% women and $46,2 \%$ men), with mean age 65.38 years $+/-2,505$ (53-79 years). During the period of 31 months, 26 implants $\left(76,9 \%\right.$ Brånemark System MkllITiUnite, Nobel Biocare ${ }^{\mathrm{TM}}$ and $23,1 \%$ TSIII SA FIXTURE, OSSTEM) with diameter from 3.3 to $4 \mathrm{~mm}$ (media of 3,76 $+-0,05$ ) and length from 8.5 to $13 \mathrm{~mm}$ (media of $11,85+-0,24$ ) were placed in 32 and 42 positions to retain mandibular overdentures. Till date all implants remained osteointegrated and so the survival rate is $100 \%$.

In 9 patients an immediate loading protocol was used $(61,5 \%$, with an insertion torque $>32 \mathrm{Ncm}$ ) with the immediate connection of the attachments and overdenture placement. In 2 of them the old prothesis was temporary adapted and in 7 a new immediate prothesis was fabricated. All prosthetic restorations were still functionally in place at the last follow-up appointment.

CONCLUSIONS: The results of this case series suggest that favorable implant survival rates can be achieved with mandibular overdentures retained by two this treatment modality. implants. An improvement in edentulous patients quality of life can be achieved with

\section{Results}

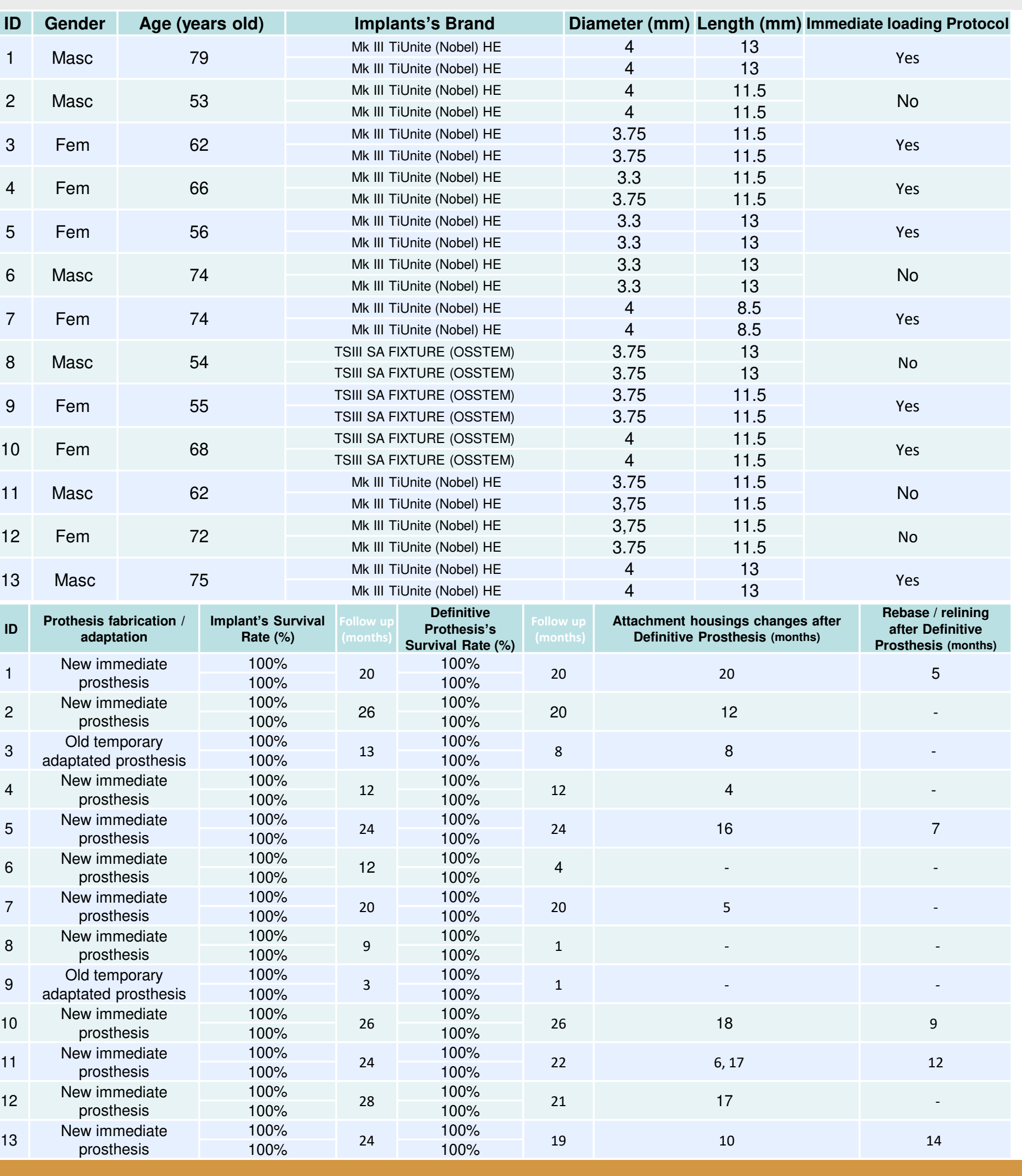

Conclusion

\section{Background and Aim}

Total edentulism remains a global health problem and its prevalence is expected to increase together with life longevity ${ }^{1}$. When compared to conventional dentures, two implant retained overdentures performs better in terms of denture stability, chewing function and in patient-centered outcomes; 2,3 when compared to multiple splinted implants also has been proved to be as effective ${ }^{4}$.

Regarding Immediate or delayed loading of mandibular overdentures, both options are well documented and validated in literature, with similar clinical outcomes ${ }^{1,5}$.

The aim of this single center retrospective case series study was to evaluate the survival rate of mandibular overdentures retained by two unsplited implants.

\begin{abstract}
Within the limitations of this case series study, the results suggest that favorable implant survival rates can be achieved with mandibular overdentures retained by two implants. An improvement in edentulous patients' quality of life can be achieved with this treatment modality.
\end{abstract}

\section{Methods and Materials}

A retrospective case series of 13 patients rehabilitated with mandibular overdentures retained by two implants, at a Post-graduation program at University of Lisbon between October 2017 and April 2019, was performed. The statistical analysis was descriptive.

Clinical Protocol: 2 implants placed at 32 and 42 positions to retain a mandibular overdenture with Locator abutment type of connection.

\section{References}

1| Salman, A, Thacker, S, Rubin, S, Dhingra, A, Ioannidou, E, Schincaglia, GP. Immediate versus delayed loading of mandibular implant-retained overdentures: A 60-month follow-up of a randomized clinical trial. $\mathrm{J}$ Clin Periodontol. 2019; 46: 863- 871; 2| Awad, M. A., Rashid, F., Feine, J. S., \& Overdenture Effectiveness Study Team Consortium (2014). The effect of mandibular 2-implant overdentures on oral health-related quality of life: An international multicentre study. Clinical Oral Implants Research, 25(1), 46-51. https $/ / /$ doi.org/10.1111/clr 12205; 3 i Heydecke, G., Thomason, J. M., Awad, M. A., Lund, J. P., \& Feine, J. S. (2008). Do mandibular implant overdentures and conventional complete dentures meet the expectations of edentulous patients? Quintessence International, 39(10), 803-809; 4| Kern, J-S, Kern, T, Wolfart, S, Heussen, N. A systematic review and meta-analysis of removable and fixed implant-supported prostheses in edentulous jaws: post-loading implan loss. Clin. Oral Impl. Res. 27, 2016, 174- 195; 5| Rignon-Bret C, Wulfman C, Hadida A, Renouard F, Gourraud PA, Naveau A. Immediate Loading of Two Unsplinted Implants in Edentulous Patients with Mandibular Overdentures: A 10-year Retrospective Review of Patients from a Previously Conducted 1-year Cohort Study. Int J Oral Maxillofac Implants. 2019 Jan/Feb;34(1):169-178. 